\title{
Aprendizaje autodirigido y su relación con el perfil valórico en estudiantes de medicina
}

\author{
EDUARDO FASCE, CRISTHIAN PÉREZ ${ }^{a}$, LILIANA ORTIZ, \\ PAULA PARRA ${ }^{\mathrm{b}}$, PILAR IBÁÑEZ ${ }^{\mathrm{c}}$, OLGA MATUS $^{\mathrm{d}}$
}

\section{Relationship between self-directed learning and value profile in Chilean medical students}

\begin{abstract}
Background: Medical education should prepare students to face a dynamic environment, through competencies that allow them to learn independently. Aim: To evaluate the relationship between self-directed learning and value profile of undergraduate first year students in a medical school in Chile. Material and Methods: SelfDirected Learning Scale and Schwartz's Values Questionnaire were applied to 235 medical students from the University of Concepción, Chile. Results: Self-direction and Security are value types that correlate directly and significantly with the overall scale and with the five subscales of Self-Directed Learning. Conclusions: In first year medical students of University of Concepcion, Chile, Self-direction and Security are values that facilitate Self-directed Learning.
\end{abstract}

(Rev Med Chile 2013; 141: 15-22).

Key words: Medical education, undergraduate; Psychology, social; Students, medical.

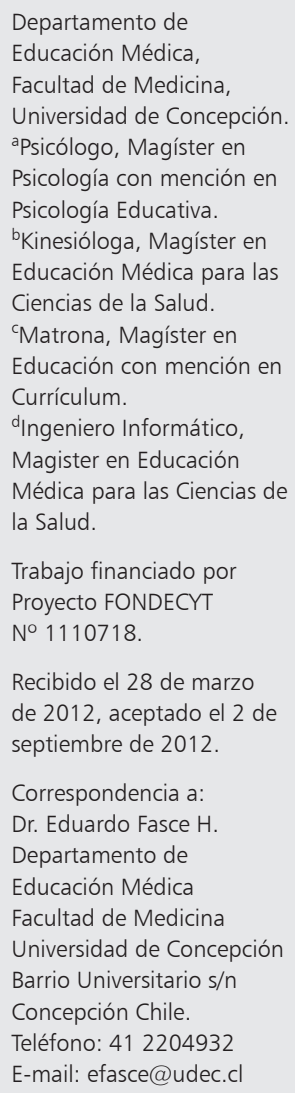

$\mathrm{E}$ 1 crecimiento exponencial del conocimiento biomédico impone la exigencia de una actualización profesional permanente. Por ello, las nuevas tendencias en educación médica subrayan el rol del estudiante en su proceso formativo, y propician cambios sustantivos en la enseñanza ${ }^{1}$.

Paralelamente, los actuales aportes de la investigación en neurociencia, y estilos y estrategias de aprendizaje, afirman la gran diversidad de las formas en que la gente aprende. La psicología cognitiva también se centra en la importancia de aprender a aprender, es decir, en el desarrollo de estrategias aplicables a cualquier tarea de aprendizaje. Tales cualidades transferibles prepararían al estudiante para formarse permanentemente ${ }^{2}$.

Una característica de los alumnos que logran éxito en este escenario, es su capacidad para autorregular su aprendizaje, favoreciendo sus resultados académicos y sus capacidades de continuar aprendiendo fuera de ambientes formales ${ }^{3}$. A esta capacidad se le ha denominado "Aprendizaje Autónomo", "Aprendizaje Autorregulado" o "Aprendizaje Autodirigido", subrayando el carácter autorreflexivo, en donde los alumnos toman la iniciativa-con o sin apoyo externo-para identificar sus necesidades y metas de aprendizaje, y seleccionar y gestionar los recursos que requieren para alcanzarlas $^{4}$, siendo el Aprendizaje Autodirigido una competencia central en las carreras de la salud ${ }^{5}$. Es por ello que el equipo de investigación del presente estudio realizó anteriormente la validación de la Escala de Aprendizaje Autodirigido de Fisher, King \& Tague en estudiantes de medicina chilenos ${ }^{6}$, con el propósito de profundizar la investigación en esta área.

La literatura ofrece numerosas comunicaciones referidas al "Aprendizaje Autodirigido". Sin embargo, las variables asociadas a éste han sido escasamente investigadas. En general, se mencio- 
nan tres grupos de éstas. Un primer grupo incluye aquellas asociadas a características intrínsecas de los alumnos, destacando la predisposición al Aprendizaje Autodirigido ${ }^{7}$, el Compromiso Académico $^{8,9}$, el nivel de conocimiento previo ${ }^{10}$, la experiencia previa $^{11}$, el Autoconcepto ${ }^{10}$, el Autocontrol $^{12}$, la capacidad de reflexión ${ }^{12,13}$ y la capacidad de autoevaluación ${ }^{11}$; un segundo grupo comprende las dependientes del proceso de enseñanza-aprendizaje ${ }^{10,11}$, la directividad de la enseñanza ${ }^{8,11}$ y la experiencia práctica ${ }^{12}$; y un tercer grupo considera las dependientes de las características del docente, incluyendo la experiencia previa en prácticas pedagógicas que estimulen la autonomía $^{8,14}$ y la experticia en el tema a enseñar ${ }^{15}$.

Además del Aprendizaje Autónomo, y basándose también en los nuevos paradigmas de los procesos de enseñanza-aprendizaje, destaca el rol de los valores en el sistema educacional actual, los que corresponden a un tipo de conocimiento social que facilita la adaptación de cada individuo a su entorno. Representan constructos motivacionales que orientan a las personas hacia metas deseables, a partir de los cuales guían sus acciones y comportamientos.

El presente estudio se basa en la importancia que otorgan al Aprendizaje Autodirigido las políticas educacionales actuales y más específicamente la Asociación de Facultades de Medicina de Chile como competencia necesaria para vivir en un mundo de constantes cambios; ; tomando en cuenta, además, a la Ley Orgánica Constitucional de Enseñanza, que considera la formación valórica como un medio que propicia la autonomía, a través del autoconocimiento, de la autoestima, la valoración positiva de la vida, el pensamiento moral autónomo y la capacidad de autorregular la conducta ${ }^{16}$, aspectos que generan el interés de analizar la posible relación entre ambas variables, a fin de aumentar la evidencia sobre los agentes que podrían predecir y/o favorecer la competencia de "Aprendizaje Autodirigido", contribuyendo de esta forma a la obtención de aprendices de por vida.

\section{Metodología}

El presente estudio fue cuantitativo, no experimental, transversal y correlacional.

Participantes: Participó la totalidad de estudiantes de primer año de medicina ingresados durante los años $2010(\mathrm{n}=117)$ y $2011(\mathrm{n}=118)$. Del total $(n=235)$, se eliminó a aquellos que tenían una tasa de omisión superior al 5\% de los ítems en los instrumentos aplicados. De esta forma, se obtuvo una muestra válida de 210 alumnos: 127 hombres (60,48\%), 77 mujeres $(36,67 \%)$ y 6 $(2,86 \%)$ participantes que no entregaron dicha información, cuyas edades oscilaron entre 17 y 29 años $(M=18,52 ; D E=1,29)$.

\section{Instrumentos}

Para evaluar la autodirección en los aprendizajes se usó la Escala de Aprendizaje Autodirigido (EPAI) de Fisher, King \& Tague, traducido al español y validado en alumnos de medicina chilenos por Fasce y cols c $^{6}$ La escala contiene 40 ítems que presentan atributos, habilidades o aspectos motivacionales característicos de los aprendices autónomos. El participante debe indicar el grado en que el ítem le representa, eligiendo entre cinco alternativas ( 1 = muy en desacuerdo; $2=$ en desacuerdo; $3=$ indeciso $; 4=$ de acuerdo y $5=$ muy de acuerdo). Tal como se detalla en el estudio de validación del instrumento ${ }^{6}$, la Escala de Aprendizaje Autodirigido (Escala General) presenta una estructura de cinco factores o subescalas que tributan al aprendizaje autodirigido: una dimensión procedimental ("Planificación del Aprendizaje"), aspectos actitudinales ("Deseo por Aprender" y "Autoconfianza"), y aspectos cognitivos vinculados a la autonomía ("Autogestión" y "Autoevaluación").

Para evaluar el perfil valórico se empleó el Cuestionario de Descripciones Valóricas de Schwartz (CDV), versión mejorada del Inventario de Valores de Schwartz que presenta un menor nivel de abstracción ${ }^{17}$, y fue probada previamente con alumnos de medicina en $2008^{18}$. En este estudio se utilizará la traducción al castellano validada por Saíz $z^{19}$. Tiene 40 ítems que presentan una breve descripción verbal de metas, aspiraciones o deseos de una persona referidos, implícitamente, a la importancia que se otorga a un tipo valórico. El sujeto debe indicar el grado en que se siente identificado por cada descripción, eligiendo entre seis alternativas $(6=$ se parece mucho a mí; $5=$ se parece a mí; $4=$ se parece algo a mí; 3 = se parece poco a mí; $2=$ no se parece a mí; $1=$ no se parece en nada a mí). El instrumento distingue diez tipos valóricos que difieren entre sí según lo que los motiva: "Autodirección": búsqueda de independencia y auto- 
confianza; "Estimulación": orientado a la emoción, la novedad y desafíos; "Hedonismo": búsqueda de placer y satisfacción; "Logro": persecución de éxito personal; "Poder": alcanzar prestigio social, dominio sobre los demás; "Seguridad": armonía y estabilidad, tanto social, interpersonal y personal; "Conformidad": control de acciones potencialmente dañinas sobre otros; "Tradición": respeto y aceptación de costumbres y aspectos culturales; "Benevolencia": valor prosocial dirigido al bienestar de los demás; "Universalismo": búsqueda del bienestar de todos y de la naturaleza.

Adicionalmente los alumnos respondieron un cuestionario sociodemográfico que permitió caracterizar la muestra.

Todos los participantes firmaron un consentimiento informado visado por el Comité de Ética de la Universidad y CONICYT.

El análisis estadístico se realizó con el software STATA SE 11.0. Para evaluar la consistencia interna de los instrumentos se usó el coeficiente alfa de Cronbach, para evaluar las relaciones bivariadas el coeficiente de correlación de Pearson y para el análisis multivariado se utilizó regresión lineal múltiple. Se consideró $\mathrm{p}<0,05$ como estadísticamente significativo.

\section{Resultados}

El análisis descriptivo de los instrumentos utilizados, junto al análisis de su confiabilidad se presentan en las Tablas 1 y 2. En éstas se observa una adecuada confiabilidad de los instrumentos.

Las correlaciones entre las puntuaciones del EPAI y el instrumento de Schwartz, se presentan en la Tabla 3. Se utilizó el coeficiente de Pearson, paramétrico, pues no presenta distorsiones en muestras sobre 40 sujetos independiente de la distribución de puntajes ${ }^{20}$. Destaca que la Autodirección y la Seguridad son los únicos tipos valóricos que correlacionan significativamente con la escala general y las cinco subescalas de aprendizaje autodirigido.

Para analizar la capacidad predictiva conjunta de los diez tipos valóricos y aislar la relación específica de cada uno de éstos se realizó un análisis multivariado mediante seis análisis de regresión lineal múltiple, uno con la Escala General de Aprendizaje Autodirigido y uno con cada Subescala (Tablas 4 a 9).

Utilizando como variable dependiente el puntaje general de la Escala de Aprendizaje Autodirigido, el conjunto de tipos valóricos hace una predicción significativa del 16,55\% de la variación de las habilidades de estudio autodirigido ( $\mathrm{p}$ $<0,001)$. Del total de tipos valóricos, sólo dos fueron predictores estadísticamente significativos: Autodirección $(\mathrm{p}<0,01)$ y Seguridad ( $<0,01)$, (Tabla 4$)$.

\section{Tabla 1. Descriptivos de la Escala de Aprendizaje Autodirigido (EPAI) en alumnos de medicina}

\begin{tabular}{|lccccc|}
\hline Escala & Alfa & M & D.E. & Mín & Máx \\
Escala general & 0,91 & 155,55 & 14,78 & 69 & 187 \\
\hline Planificación del aprendizaje & 0,85 & 38,06 & 6,17 & 22 & 50 \\
\hline Deseo de aprender & 0,81 & 26,14 & 3,21 & 8 & 30 \\
\hline Autoconfianza & 0,79 & 38,09 & 4,24 & 14 & 45 \\
\hline Autogestión & 0,71 & 38,31 & 3,75 & 13 & 45 \\
Autoevaluación & 0,66 & 14,95 & 2,61 & 8 & 20 \\
\hline
\end{tabular}

$\mathrm{n}=210 ;$ Alfa = coeficiente alfa de Cronbach; $M=$ media; D.E. = desviación estándar; Min = valor mínimo; Máx = valor máximo.

Tabla 2. Descriptivos del Cuestionario de Tipos Valóricos en alumnos de medicina

\begin{tabular}{|lccccc|}
\hline Tipo valórico & Alfa & M & D.E. & Mín & Máx \\
Conformidad & 0,61 & 17,92 & 3,39 & 5 & 24 \\
Tradición & 0,43 & 15,48 & 3,36 & 5 & 23 \\
Benevolencia & 0,56 & 20,85 & 2,30 & 12 & 24 \\
Universalismo & 0,73 & 30,97 & 3,75 & 18 & 36 \\
Autodirección & 0,51 & 20,71 & 2,27 & 12 & 24 \\
Estimulación & 0,71 & 18,83 & 2,72 & 6 & 18 \\
Hedonismo & 0,84 & 14,15 & 3,05 & 3 & 18 \\
Logro & 0,83 & 16,78 & 4,11 & 6 & 24 \\
Poder & 0,67 & 9,94 & 2,96 & 3 & 17 \\
Seguridad & 0,70 & 21,20 & 4,64 & 7 & 30 \\
\hline
\end{tabular}

$\mathrm{n}=210$; Alfa = coeficiente alfa de Cronbach; $M=$ media; D.E. = desviación estándar; Min = valor mínimo; Máx = valor máximo. 
Aprendizaje autodirigido y perfil valórico en alumnos de medicina - E. Fasce et al

Tabla 3. Correlación de los niveles de aprendizaje autodirigido con el perfil valórico de los alumnos de medicina

\begin{tabular}{|llccccc|}
\hline $\begin{array}{l}\text { Perfil } \\
\text { valórico }\end{array}$ & $\begin{array}{c}\text { Escala } \\
\text { general }\end{array}$ & $\begin{array}{c}\text { Planificación del } \\
\text { aprendizaje }\end{array}$ & $\begin{array}{c}\text { Deseo de } \\
\text { aprender }\end{array}$ & Autoconfianza & Autogestión & Autoevaluación \\
\hline Conformidad & 0,13 & $0,26^{* *}$ & 0,13 & $-0,03$ & $-0,01$ & 0,05 \\
\hline Tradición & $0,14^{*}$ & $0,15^{*}$ & 0,10 & 0,04 & 0,10 & 0,12 \\
\hline Benevolencia & $0,26^{* * *}$ & 0,17 & $0,21^{* *}$ & 0,23 & $0,19^{* *}$ & $0,17^{*}$ \\
Universalismo & $0,24^{* *}$ & 0,10 & $0,32^{* * *}$ & $0,15^{*}$ & $0,18^{*}$ & $0,21^{* *}$ \\
\hline Autodirección & $0,34^{* * *}$ & $0,16^{*}$ & $0,28^{* * *}$ & $0,36^{* *}$ & $0,25^{* * *}$ & $0,28^{* * *}$ \\
\hline Estimulación & $0,17^{*}$ & 0,01 & $0,17^{*}$ & $0,23^{* * *}$ & $0,16^{*}$ & 0,08 \\
\hline Hedonismo & 0,09 & $-0,01$ & 0,06 & $0,17^{*}$ & 0,11 & 0,02 \\
\hline Logro & $0,04^{*}$ & 0,00 & $-0,06$ & $0,17^{*}$ & $-0,01$ & 0,02 \\
\hline Poder & $0,14^{*}$ & 0,10 & $-0,04$ & $0,23^{* * *}$ & 0,06 & $0,14^{*}$ \\
\hline Seguridad & $0,32^{* * *}$ & $0,31^{* * *}$ & $0,19^{* *}$ & $0,22^{* *}$ & $0,15^{*}$ & $0,28^{* * *}$ \\
\hline
\end{tabular}

$\mathrm{n}=210 ;{ }^{*} \mathrm{p}<0,05 ;{ }^{* *} \mathrm{p}<0,01 ;{ }^{* * *} \mathrm{p}<0,001$.

Tabla 4. Resultados de la regresión lineal múltiple para la Escala General de Aprendizaje Autodirigido

\begin{tabular}{|c|c|c|c|c|}
\hline & B & EE & $\beta$ & sr2 \\
\hline Constante & 88,58 & & & \\
\hline Conformidad & $-0,26$ & 0,38 & $-0,06$ & 0,00 \\
\hline Tradición & 0,23 & 0,34 & 0,05 & 0,00 \\
\hline Benevolencia & 0,93 & 0,52 & 0,15 & 0,01 \\
\hline Universalismo & $-0,00$ & 0,32 & $-0,00$ & 0,00 \\
\hline Autodirección & $1,73^{* *}$ & 0,54 & 0,26 & 0,04 \\
\hline Estimulación & $-0,32$ & 0,52 & $-0,06$ & 0,00 \\
\hline Hedonismo & 0,17 & 0,39 & 0,03 & 0,00 \\
\hline Logro & $-0,23$ & 0,32 & $-0,07$ & 0,00 \\
\hline Poder & 0,23 & 0,45 & 0,05 & 0,00 \\
\hline Seguridad & $0,79^{* *}$ & 0,26 & 0,25 & 0,04 \\
\hline \multicolumn{5}{|c|}{$R^{2}=0,21^{* * *} ; R^{2}$ ajustado $=0,17$} \\
\hline
\end{tabular}

$\mathrm{B}=$ coeficiente de regresión no estandarizado; $\mathrm{EE}=$ error estándar; $\beta=$ coeficiente de regresión estandarizado; $\mathrm{sr}^{2}=$ cuadrado de correlaciones semiparciales; $R^{2}=$ coeficiente de determinación; $R^{2}$ ajustado = coeficiente de determinación ajustado. $\mathrm{n}=210 ;{ }^{*} \mathrm{p}<0,05 ;{ }^{* *} \mathrm{p}<0,01 ;{ }^{* *} \mathrm{p}<0,001$.

Para la Subescala de Planificación del Aprendizaje, los tipos valóricos en su conjunto también fueron estadísticamente significativos, logrando una predicción de $9,78 \%$ de la variación de las habilidades de planificación $(\mathrm{p}<0,001)$. Indi-
Tabla 5. Resultados de la regresión lineal múltiple para la Subescala de Planificación del Aprendizaje

\begin{tabular}{|lcccc|}
\hline & B & EE & $\boldsymbol{\beta}$ & sr2 \\
Constante & 21,72 & & & \\
Conformidad & 0,26 & 0,16 & 0,14 & 0,01 \\
\hline Tradición & $-0,03$ & 0,15 & $-0,02$ & 0,00 \\
\hline Benevolencia & 0,36 & 0,23 & 0,14 & 0,01 \\
\hline Universalismo & $-0,21$ & 0,14 & $-0,13$ & 0,01 \\
\hline Autodirección & 0,40 & 0,23 & 0,15 & 0,01 \\
Estimulación & $-0,10$ & 0,22 & $-0,05$ & 0,00 \\
\hline Hedonismo & $-0,04$ & 0,17 & $-0,02$ & 0,00 \\
\hline Logro & $-0,15$ & 0,14 & $-0,10$ & 0,01 \\
\hline Poder & 0,11 & 0,19 & 0,05 & 0,00 \\
\hline Seguridad & $0,30^{*}$ & 0,11 & 0,22 & 0,03 \\
\hline$R^{2}=0,14^{* * *} R^{2}$ ajustado $=0,10$ & & \\
\hline
\end{tabular}

$\mathrm{B}=$ coeficiente de regresión no estandarizado; $\mathrm{EE}=$ error estándar; $\beta=$ coeficiente de regresión estandarizado; $s r^{2}=$ cuadrado de correlaciones semiparciales; $R^{2}=$ coeficiente de determinación; $R^{2}$ ajustado = coeficiente de determinación ajustado. $\mathrm{n}=210 ;{ }^{*} \mathrm{p}<0,05 ;{ }^{* *} \mathrm{p}<0,01 ;{ }^{* * *} \mathrm{p}<0,001$

vidualmente, sólo el tipo valórico Seguridad fue estadísticamente significativo ( $\mathrm{p}<0,05)$, (Tabla 5).

Para la Subescala de Deseo por Aprender, la escala de valores también resultó estadísticamente significativa logrando una predicción de 16,81\% 
Aprendizaje autodirigido y perfil valórico en alumnos de medicina - E. Fasce et al

Tabla 6. Resultados de la regresión lineal múltiple para la Subescala de Deseo de Aprender

\begin{tabular}{|lcccc|}
\hline & B & EE & $\boldsymbol{\beta}$ & sr2 \\
Constante & 13,47 & & & \\
Conformidad & 0,06 & 0,08 & 0,06 & 0,00 \\
Tradición & 0,00 & 0,08 & 0,00 & 0,00 \\
Benevolencia & $-0,02$ & 0,12 & $-0,01$ & 0,00 \\
Universalismo & $0,15^{*}$ & 0,07 & 0,17 & 0,02 \\
Autodirección & $0,31^{*}$ & 0,12 & 0,22 & 0,00 \\
Estimulación & 0,05 & 0,12 & 0,05 & 0,00 \\
Hedonismo & 0,03 & 0,09 & 0,03 & 0,00 \\
Logro & $-0,01$ & 0,07 & $-0,01$ & 0,00 \\
Poder & $-0,16$ & 0,10 & $-0,15$ & 0,01 \\
\hline Seguridad & 0,07 & 0,06 & 0,10 & 0,01 \\
\hline$R^{2}=0,20 * * *$ & $R^{2}$ ajustado $=0,17$ & & \\
\hline
\end{tabular}

$\mathrm{B}=$ coeficiente de regresión no estandarizado; $\mathrm{EE}=$ error estándar; $\beta=$ coeficiente de regresión estandarizado; $\mathrm{sr}^{2}=$ cuadrado de correlaciones semiparciales; $R^{2}=$ coeficiente de determinación; $R^{2}$ ajustado = coeficiente de determinación ajustado. $\mathrm{n}=210 ;{ }^{*} \mathrm{p}<0,05 ;{ }^{* *} \mathrm{p}<0,01 ;{ }^{* * *} \mathrm{p}<0,001$.

de su variación $(\mathrm{p}<0,001)$. Individualmente, los tipos valóricos que resultaron ser predictores estadísticamente significativos fueron la Autodirección $(\mathrm{p}<0,05)$ y el Universalismo $(\mathrm{p}<0,05)$, (Tabla 6).

Para la Subescala de Autoconfianza, el conjunto de valores realizó una predicción estadísticamente significativa del $16,56 \%$ de esta variable $(\mathrm{p}<0,001)$. Los predictores estadísticamente significativos a nivel individual fueron los tipos valóricos de Conformidad ( $\mathrm{p}<0,05)$, de Benevolencia $(\mathrm{p}<0,05)$, de Autodirección $(\mathrm{p}<0,01) \mathrm{y}$ de Seguridad $(\mathrm{p}<0,05)$, (Tabla 7).

Para la Subescala de Autogestión la escala de valores resultó estadísticamente significativa, prediciendo el $6,27 \%$ de ésta $(p<0,05)$. Individualmente, el único predictor estadísticamente significativo fue la Autodirección ( $\mathrm{p}<0,05)$, (Tabla 8).

Finalmente, para la Subescala de Autoevaluación la escala valórica logró una predicción estadísticamente significativa de $12,06 \%$ de la variable $(\mathrm{p}<0,001)$. Individualmente, los tipos valóricos de Conformidad $(\mathrm{p}<0,05)$, Autodirección $(\mathrm{p}<0,05)$, y Seguridad $(\mathrm{p}<0,01)$ resultaron predictores estadísticamente significativos, (Tabla 9).
Tabla 7. Resultados de la regresión lineal múltiple para la Subescala de Autoconfianza

\begin{tabular}{|lcccc|}
\hline & B & EE & $\boldsymbol{\beta}$ & sr2 \\
Constante & 20,07 & & & \\
Conformidad & $-0,26^{*}$ & 0,11 & $-0,21$ & 0,02 \\
\hline Tradición & 0,07 & 0,10 & 0,06 & 0,00 \\
Benevolencia & $0,34^{*}$ & 0,15 & 0,18 & 0,02 \\
\hline Universalismo & $-0,02$ & 0,09 & $-0,01$ & 0,00 \\
\hline Autodirección & $0,45^{* *}$ & 0,15 & 0,24 & 0,03 \\
\hline Estimulación & $-0,09$ & 0,15 & $-0,06$ & 0,00 \\
\hline Hedonismo & 0,08 & 0,11 & 0,05 & 0,00 \\
\hline Logro & 0,04 & 0,09 & 0,04 & 0,00 \\
\hline Poder & 0,15 & 0,13 & 0,11 & 0,01 \\
\hline Seguridad & $0,18^{*}$ & 0,08 & 0,19 & 0,02 \\
\hline$R^{2}=0,21 * * * ;$ & $R^{2}$ ajustado $=0,17$ & & \\
\hline
\end{tabular}

$\mathrm{B}=$ coeficiente de regresión no estandarizado; $\mathrm{EE}=$ error estándar; $\beta$ = coeficiente de regresión estandarizado; $\mathrm{sr}^{2}=$ cuadrado de correlaciones semiparciales; $R^{2}=$ coeficiente de determinación; $R^{2}$ ajustado = coeficiente de determinación ajustado. $\mathrm{n}=210 ;{ }^{*} \mathrm{p}<0,05 ;{ }^{* *} \mathrm{p}<0,01 ;{ }^{* *} \mathrm{p}<0,001$.

Tabla 8. Resultados de la regresión lineal múltiple para la Subescala de Autogestión

\begin{tabular}{|lcccc|}
\hline & B & EE & B & sr2 \\
Constante & 26,82 & & & \\
Conformidad & $-0,17$ & 0,10 & $-0,16$ & 0,01 \\
\hline Tradición & 0,12 & 0,09 & 0,11 & 0,01 \\
Benevolencia & 0,15 & 0,14 & 0,09 & 0,00 \\
Universalismo & 0,03 & 0,09 & 0,03 & 0,00 \\
Autodirección & $0,32^{*}$ & 0,14 & 0,19 & 0,02 \\
\hline Estimulación & $-0,06$ & 0,14 & $-0,04$ & 0,00 \\
\hline Hedonismo & 0,11 & 0,10 & 0,09 & 0,00 \\
\hline Logro & $-0,07$ & 0,08 & $-0,07$ & 0,00 \\
\hline Poder & 0,03 & 0,12 & 0,02 & 0,00 \\
\hline Seguridad & 0,11 & 0,07 & 0,13 & 0,01 \\
\hline$R^{2}=0,11 * ; R^{2}$ ajustado $=0,06$. & & \\
\hline
\end{tabular}

$B=$ coeficiente de regresión no estandarizado; $E E=$ error estándar; $\beta=$ coeficiente de regresión estandarizado; $\mathrm{sr}^{2}=$ cuadrado de correlaciones semiparciales; $R^{2}=$ coeficiente de determinación; $R^{2}$ ajustado = coeficiente de determinación ajustado. $\mathrm{n}=210$; ${ }^{*} \mathrm{p}<0,05 ;{ }^{* *} \mathrm{p}<0,01 ;{ }^{* *} \mathrm{p}<0,001$. 
Tabla 9. Resultados de la regresión lineal múltiple para la Subescala de Autoevaluación

\begin{tabular}{|lcccc|}
\hline & B & EE & B & sr2 \\
Constante & 6,50 & & & \\
Conformidad & $-0,15^{*}$ & 0,07 & $-0,19$ & 0,02 \\
\hline Tradición & 0,07 & 0,06 & 0,08 & 0,00 \\
Benevolencia & 0,10 & 0,09 & 0,09 & 0,00 \\
Universalismo & 0,04 & 0,06 & 0,06 & 0,00 \\
Autodirección & $0,25^{*}$ & 0,10 & 0,22 & 0,03 \\
Estimulación & $-0,12$ & 0,09 & $-0,13$ & 0,00 \\
Hedonismo & $-0,00$ & 0,07 & $-0,00$ & 0,00 \\
Logro & $-0,05$ & 0,06 & $-0,08$ & 0,00 \\
\hline Poder & 0,10 & 0,08 & 0,11 & 0,01 \\
\hline Seguridad & $0,14^{* *}$ & 0,05 & 0,25 & 0,04 \\
\hline$R^{2}=0,16^{* * *} ; R^{2}$ ajustado $=0,12$ & & \\
\hline
\end{tabular}

$\mathrm{B}=$ coeficiente de regresión no estandarizado; $\mathrm{EE}=$ error estándar; $\beta=$ coeficiente de regresión estandarizado; $s r^{2}=$ cuadrado de correlaciones semiparciales; $R^{2}=$ coeficiente de determinación; $R^{2}$ ajustado $=$ coeficiente de determinación ajustado. $\mathrm{n}=210 ;{ }^{*} \mathrm{p}<0,05 ;{ }^{* *} \mathrm{p}<0,01 ;{ }^{* * *} \mathrm{p}<0,001$.

\section{Discusión}

Considerando que la revisión bibliográfica no permitió identificar publicaciones que relacionen valores con aprendizaje autodirigido, la discusión estará centrada en el análisis de los resultados obtenidos en este estudio.

Los principales resultados obtenidos comprueban una relación significativa entre los valores del modelo de Schwartz y el Aprendizaje Autodirigido en estudiantes de primer año de medicina, siendo los tipos valóricos de Autodirección y Seguridad aquéllos que individualmente presentaron correlaciones estadísticamente significativas, tanto con la Escala General de Aprendizaje Autodirigido como con sus cinco subescalas.

Es así como en el análisis bivariado, la $\mathrm{Au}$ todirección, caracterizada por la autoconfianza, la búsqueda de independencia y la gratificación basada en la capacidad de toma de decisiones, se correlaciona positivamente con todas las puntuaciones del Aprendizaje Autodirigido. Lo mismo ocurre con el valor Seguridad, orientado a la búsqueda de la armonía y estabilidad tanto de sí mismo como de las relaciones interpersonales, indicando que los sujetos toman mayor protagonismo en sus procesos de aprendizaje al tener un mayor control sobre ellos, dependiendo en menor grado del apoyo externo. Una mayor dependencia externa (docentes, recursos, etc.) determinaría una menor seguridad en obtener buenos resultados, cuyo logro sería más dependiente del propio esfuerzo personal.

En concordancia con lo anterior, en el análisis multivariado, el tipo valórico de Autodirección aparece como el predictor individual más importante, ya sea con los puntajes de la Escala General de Aprendizaje Autodirigido y de cuatro de las cinco subescalas: Deseo de Aprender, Autoconfianza, Autogestión y Autoevaluación. Esto muestra que los sujetos que valoran más dirigir sus propias acciones también presentan una mayor orientación a aprender de manera autónoma. Ello es conceptualmente esperable, así como el hecho que para la única subescala donde no es un predictor estadísticamente significativo, esto es Planificación del Aprendizaje, van implícitas habilidades únicamente procedimentales, carentes del fuerte componente actitudinal de las anteriores.

El segundo predictor más importante es el tipo valórico de Seguridad, que fue estadísticamente significativo para la Escala General y para tres subescalas: Planificación del Aprendizaje, Autoconfianza y Autoevaluación. La necesidad de un entorno estable y controlable, que sería característico de los sujetos orientados por este perfil valórico, podría llevarlos a ser más precavidos y detallistas en sus procesos de aprendizaje, siendo más planificados, así como llevarlos a desarrollar más actividades, e incluso habilidades, para supervisar dichos procesos, evaluándose a sí mismos con mayor frecuencia.

También el tipo valórico de Universalismo mostró ser un predictor significativo del Deseo de Aprender y la Benevolencia de la Autoconfianza. Esto puede deberse a que un sujeto cuya meta es promover el bienestar social y la armonía del ambiente se esforzaría en alcanzar los aprendizajes necesarios para tal logro (motivación intrínseca), presentando un mayor interés por sus aprendizajes. En oposición a ello, quien valora más su propio bienestar, en desmedro de otros, exhibiría una disposición más orientada a obtener beneficios personales tangibles (buenas calificaciones, reconocimiento externo), en lugar de aprendizaje profundo (motivación extrínseca). Asimismo, 
quien desea el bienestar de los demás, requiere de más recursos para lograr su meta por lo que el aprendizaje se convierte en una necesidad valiosa en sí misma.

La correlación positiva entre Deseo por Aprender y los tipos valóricos de Autodirección y Universalismo indican que quienes valoran más la búsqueda independiente de sus objetivos y el bienestar de todos los demás, son también aquellos que se muestran más motivados por lograr nuevos aprendizajes

Un caso especial es el tipo valórico Conformidad, que fue un predictor estadísticamente significativo de la Autoconfianza y la Autoevaluación. De acuerdo a Schwartz, este valor se expresa en la capacidad de restringir eventuales impulsos que puedan dañar a otros o alterar las expectativas sociales, condición que es más alcanzable en quienes se autoevalúen y posean mayor confianza en sí mismos. De esta forma, quienes restringen menos sus acciones para alterar las expectativas sociales, quienes se preocupan más por el bienestar del otro, quienes valoran más la independencia en sus acciones y decisiones y quienes prefieren la armonía y estabilidad, son también quienes tienen mayor confianza en poder realizar exitosamente sus actividades de aprendizaje.

Como era esperable, se comprobó correlación significativa entre la subescala de Autogestión y la de Autodirección, mostrando que quienes valoran más la autonomía presentan mayores habilidades para administrar sus procesos de aprendizaje.

Finalmente, la subescala de Autoevaluación se correlacionó con los tipos valóricos de Conformidad, Autodirección y Seguridad, demostrando que quienes se muestran más capaces de supervisar sus procesos de aprendizaje valoran más actuar y decidir independientemente, otorgando preferencia a la estabilidad.

Los tipos valóricos en su conjunto explican una quinta parte del aprendizaje autodirigido. Esto es esperable, pues la cosmovisión del sujeto no guarda una relación directa con las actitudes puntuales ante actividades y/u objetos específicos de su vida.

Sin embargo, esto muestra que más allá de los conocimientos académicamente relevantes, las actitudes hacia los estudios y las estrategias de aprendizaje, existen ciertos valores globales a los que debe adscribir una persona para llegar a constituirse en un aprendiz autónomo. Esto implica que sujetos que son dependientes en otras áreas de su vida o que presentan altos niveles de pasividad ante su ambiente, tienen menores posibilidades de constituirse en sujetos que guíen de manera independiente sus estudios. Por el contrario, escuelas de medicina que motiven que los estudiantes tomen sus decisiones, se responsabilicen de sus acciones y valoren las consecuencias positivas del aprendizaje, determinaría mayores posibilidades de formar aprendices independientes.

Los resultados permiten identificar variables potencialmente útiles en el diagnóstico y selección de los estudiantes. No obstante, es mejor considerarlos como una guía para la formulación de innovaciones que fomenten el aprendizaje autodirigido en la formación médica, entendiendo que el desarrollo de la autonomía y sus valores asociados es una responsabilidad que las escuelas de medicina debieran asumir.

\section{Referencias}

1. Pérez C, Parra P, Ortiz L, Fasce E. Variables personales y académicas asociadas al aprendizaje autodirigido en la educación médica. Rev Cienc Salud 2010; 7 (2): 152-60.

2. Knowles M. Self-Directed Learning: A Guide for Learners and Teachers. New York: Cambridge Book Company; 1975.

3. Gibbons M. The Self-Directed Learning Handbook, John Wiley \& Sons, 2002, pág. 1-2.

4. Kocaman G, Dicle A, Ugur A. A longitudinal Analysis of the Self-Directed Learning Readiness level of Nursing Students Enrolled in a Problem-Based Curriculum. J Nurs Educ 2009; 48: 286-90.

5. Parra P. El Aprendizaje Autodirigido en el Contexto de la Educación Médica. Rev Cienc Salud 2010; 7 (2): 146-51.

6. Fasce E, Pérez C, Ortiz L, Parra P, Matus O. Estructura Factorial y Confiabilidad de la Escala de Aprendizaje Autodirigido de Fisher, King \& Tague En Alumnos De Medicina Chilenos. Rev Med Chile 2011; 139: 1428-34.

7. Hoban JD, Lawson S, Mazmanian PL, Best AM, Seibel H R. The Self-Directed Learning Readiness Scale: A factor analysis study. Med Educ 2005; 39 (4): 370-9.

8. MacDougall M. Ten tips for promoting autonomous learning and effective engagement in teaching of statistics to undergraduate medical students involved in short-term research projects. JAQM 2008; 3 (3): 223-40.

9. Parra P. Relación entre el nivel de engagement y el rendimiento académico teórico/práctico. Rev Educ Cienc Salud 2010; 7 (1): 57-63.

10. Fisher M, King J, Tague G. Development of a selfdirected learning readiness scale for nursing education. 
Nurse Educ Today 2001; 21 (7): 516-25.

11. Grevenson GC, Spencer JA. Self-directed learning - the importance of concepts and contexts. Med Educ 2005; 39 (4): 348-9.

12. Lindh M, Hugo J. Students' reflections on self-directed learning using patient studies in a Masters programme in family medicine in South Africa. Educ Prim Care 2005; 16 (4): 474-81.

13. Roberts C, Stark P. Readiness for self-directed change in professional behaviors: Factorial validation of the Selfreflection and Insight Scale. Med Educ 2008; 42 (11): 1054-63.

14. Pérez C. Diez consejos para promover el aprendizaje autónomo y el compromiso efectivo al enseñar contenidos complejos. Rev Educ Cienc Salud 2010; 7 (1): 50-6.

15. O'Shea E. Self-directed learning in nurse education: a review of the literature. J Adv Nurs 2005; 43 (1): 62-70.

16. Carrasco E, Osses S. Estudio del Perfil Valórico. El Caso de los Estudiantes de Primer Nivel de la Carrera de Obstetricia y Puericultura de la Facultad de Medicina de la Universidad de la Frontera, Temuco, Chile Estud Pedagóg 2005; 31 (1): 7-32.

17. Castro A, Nader M. La evaluación de los valores humanos mediante el Portrait Values Questionnaire de Schwartz. Interdisciplinaria 2006; 23 (2): 155-74.

18. Navarro G, Cottin I, Fasce E, Pérez C. Valores y orientación social en estudiantes de medicina de primero y séptimo año de la Universidad de Concepción. Rev Educ Cienc Salud 2009; 6 (1): 42-8.

19. Saiz J. Valores en estudiantes universitarios mapuches: una visión transcultural de su contenido, estructura y jerarquía. Tesis para optar al grado de Doctor en Psicología. Universidad Católica de Chile, Santiago, 2003. www. uc.cl/comunicaciones/site/noticias/ficha/pub877.html

20. Pardo A, Ruiz MA, San Martín, R. Análisis de datos en ciencias sociales y de la salud I. Madrid: Síntesis; 2010. 